Frontiers in Optics 2006 The 90th OSA Annual Meeting Laser Science XXII Optical Fabrication and Testing Topical Meeting Organic Photonics and Electronics Topical Meeting

October 8-12, 2006 Rochester, New York, USA
Table of Contents

Technical Session Abstracts

Agenda of Sessions

Committees

Key to Authors and Presiders

www.frontiersinoptics.org www.osa.org/oft www.osa.org/ope

\section{Citation Information}

Optical Society of America

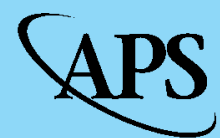

AMERICAN PIIYSICAL SOCIETY 


\section{Citation Information}

Papers submitted to the FiO/LS/OF\&T/OPE meetings should be considered archival and may be cited in other publications. As an FiO/LS/OF\&T/OPE submission is a submission to a technical conference and an archival publication, $\mathrm{FiO} / \mathrm{LS} / \mathrm{OF} \& \mathrm{~T} / \mathrm{OPE}$ papers may not appear in other publications or journals.

Proper citation for the Technical Digest CD-ROM should be written in the following format:

Name of Author (s), "Title of Paper," in Frontiers in Optics, Laser Science, Optical Fabrication and Testing and Organic Photonics and Electronics 2006 (Optical Society of America, Washington, DC, 2006), presentation number. 


\title{
Tolerance Analysis of PCB-integrated Optical Interconnects
}

\author{
Nina Hendrickx ${ }^{1}$, Jürgen Van Erps ${ }^{2}$, Hugo Thienpont ${ }^{2}$, Peter Van Daele ${ }^{1}$ \\ ${ }^{1}$ INTEC-TFCG Microsystems, Ghent University, ${ }^{2}$ TONA, Vrije Universiteit Brussel \\ nina.hendrickx@intec.ugent.be
}

\begin{abstract}
: the alignment tolerances between the different building blocks of an optical interconnection are studied both numerically and experimentally. Laser ablation is used for the structuring of polymer optical layers integrated on a printed circuit board.

(C) 2006 Optical Society of America

OCIS codes: (350.3390) Laser materials processing; (220.0220) Optical design and fabrication
\end{abstract}

Laser ablation is a versatile technology that can be used for the micro-structuring of polymer optical layers integrated on a printed circuit board. Both multimode waveguides and coupling structures are ablated in a highly crosslinked acrylate-based polymer material. The material shows excellent optical, thermal and planarization properties and is compatible with standard PCB manufacturing and soldering processes. Arrays of $50 \times 50 \mu \mathrm{m}^{2}$ multimode waveguides are ablated into the polymer layer on a pitch of $125 \mu \mathrm{m}$ to guide the light in the plane of the optical layer. Metallized $45^{\circ}$ micro-mirrors are used for out-of-plane coupling. KrF excimer laser (248nm) is used for the definition of both the waveguides and micro-mirrors. During the processing the sample is placed on a computer controlled translation stage, which has an accuracy of $1 \mu \mathrm{m}$. The excimer laser beam can be tilted; the sample can thus stay horizontally on the translation stage during the processing of the $45^{\circ}$ micro-mirrors. The alignment between elements in a multilayer structure is achieved by using alignment marks, placed on the substrate.

Numerical simulations are performed with a non-sequential ray tracing program (ASAP) to determine the

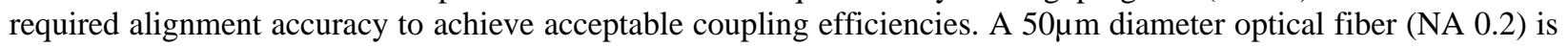
used to couple the light into the waveguides; a $100 \mu \mathrm{m}$ diameter optical fiber (NA 0.29) is used to detect the outcoupled energy. No scattering losses are taken into account. During the ablation there is always a certain degree of tapering. The cross-section of the waveguides is therefore trapezoidal instead of square. This turns out to have a very limited influence on the coupling efficiency of the optical waveguide. The influence on the coupling efficiency of a deviation of the desired $45^{\circ}$ angle of the micro-mirror is investigated. The results show that a small deviation of the angle has a limited influence on the coupling efficiency because of the presence of the metal film. The tolerance for a mechanical misalignment of the outcoupling fiber with respect to the out-of-plane turning mirror has also been evaluated. The simulation results indicate that a misalignment of $+-40 \mu \mathrm{m}$ gives an excess loss of $1 \mathrm{~dB}$. A two layer optical structure, which contains two waveguiding layers, is also investigated. A $\pm 10 \mu \mathrm{m}$ misalignment between the waveguides in the top and bottom layer decreases the coupling efficiency towards an array of $100 \mu \mathrm{m}$ diameter circular detectors (pitch $125 \mu \mathrm{m}$ ) by $1 \mathrm{~dB}$.

Experimental results indicate that the alignment accuracy between the ablated waveguides in top and bottom optical layer is $\leq 5 \mu \mathrm{m}$, which is in accordance with the simulation results. The $45^{\circ}$ angle of the micro-mirror has been confirmed with a stylus profiler (Dektak). Surface roughness measurements are performed with a non-contact optical profiler (Wyko NT-2000). These measurements indicate that the sidewalls of the waveguides have an average RMS surface roughness of 35nm; the micro-mirror has an average RMS surface roughness of 53nm.
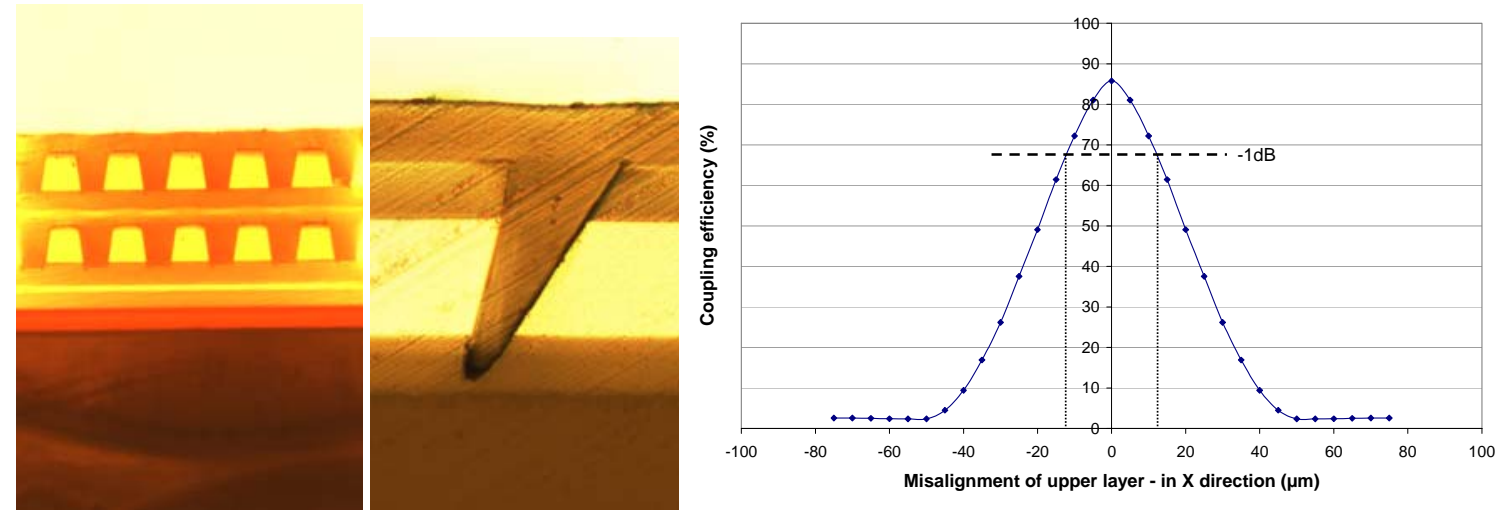

Figure 1: two layer waveguide structure; metallised $45^{\circ}$ micro-mirror; simulated alignment tolerance for two layer waveguide structure. 